Supplementary Information for:

\title{
Molecular Assembly of Surfactant Mixtures in Oil-Swollen Micelles: Implications for High Salinity Colloidal Stability
}

Hsieh Chen, ${ }^{1, *}$ Ayrat Gizzatov, ${ }^{1}$ and Amr I. Abdel-Fattah ${ }^{2}$

${ }^{1}$ Aramco Services Company: Aramco Research Center-Boston, 400 Technology Square, Cambridge, Massachusetts 02139, United States

${ }^{2}$ EXPEC ARC, Reservoir Engineering Technology Division, Saudi Aramco, Dhahran 31311, Saudi

Arabia

"hsieh.chen@aramcoservices.com 


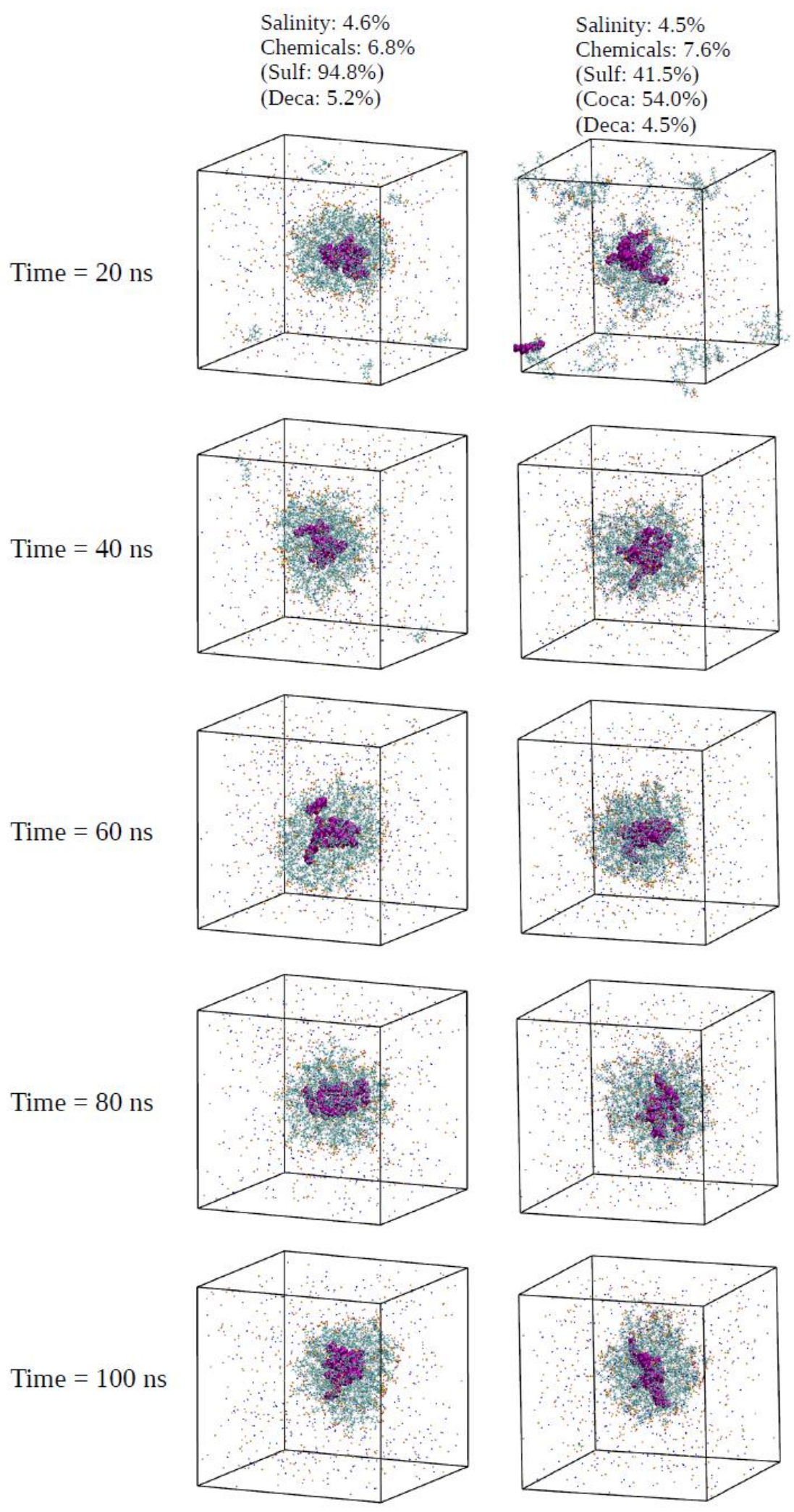

Fig. S1. Representative simulation snapshots over time for the formation of micelles. Note the stable micelles were formed around time $=40 \mathrm{~ns}$. 


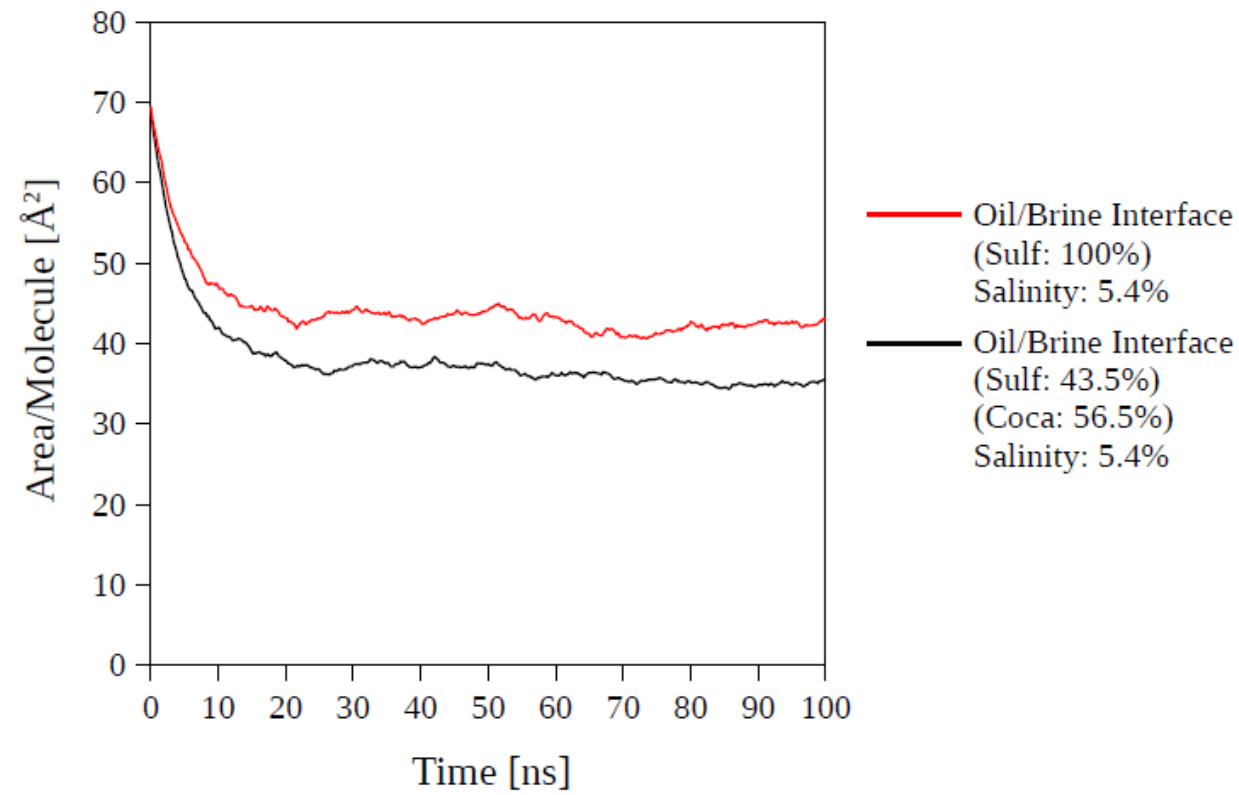

Fig. S2. Representative area per molecule (area/molecule) for the surfactants at oil/brine interfaces as a function of simulation time. Note the area/molecule plateaued around time $=30 \mathrm{~ns}$. 
Table S1. Summary of the aggregation numbers of the micelles as observed from the simulation snapshots shown in Fig. 4.

\begin{tabular}{|c|c|c|c|}
\hline $\mathbf{A}$ & B & C & D \\
\hline $\begin{array}{l}\frac{\text { Micelle } 1}{\text { Sulf }=9} \\
\text { Deca }=0\end{array}$ & $\begin{array}{l}\frac{\text { Micelle } 1}{\text { Sulf }=15} \\
\text { Deca }=0\end{array}$ & $\begin{array}{l}\frac{\text { Micelle } 1}{\text { Sulf }=44} \\
\text { Deca }=6\end{array}$ & $\begin{array}{l}\text { Micelle 1 } \\
\text { Sulf }=16 \\
\text { Deca }=2\end{array}$ \\
\hline $\begin{array}{l}\frac{\text { Micelle 2 }}{\text { Sulf }=14} \\
\text { Deca }=0\end{array}$ & $\begin{array}{l}\frac{\text { Micelle } 2}{\text { Sulf }=20} \\
\text { Deca }=3\end{array}$ & $\begin{array}{l}\text { Micelle } 2 \\
\text { Sulf }=36 \\
\text { Deca }=4\end{array}$ & $\begin{array}{l}\text { Micelle 2 } \\
\text { Sulf }=20 \\
\text { Deca }=5\end{array}$ \\
\hline $\begin{array}{l}\frac{\text { Micelle } 3}{\text { Sulf }=26} \\
\text { Deca }=0\end{array}$ & $\begin{array}{l}\frac{\text { Micelle } 3}{\text { Sulf }=45} \\
\text { Deca }=2\end{array}$ & & $\begin{array}{l}\text { Micelle } 3 \\
\text { Sulf }=44 \\
\text { Deca }=13\end{array}$ \\
\hline $\begin{array}{l}\frac{\text { Micelle } 4}{\text { Sulf }=31} \\
\text { Deca }=0\end{array}$ & & & \\
\hline $\mathbf{E}$ & $\mathbf{F}$ & G & H \\
\hline $\begin{array}{c}\text { Micelle 1 } \\
\text { Sulf }=22 \\
\text { Coca }=21 \\
\text { Deca }=0\end{array}$ & $\begin{array}{c}\text { Micelle 1 } \\
\text { Sulf }=19 \\
\text { Coca }=23 \\
\text { Deca }=3\end{array}$ & $\begin{array}{c}\text { Micelle } 1 \\
\text { Sulf }=10 \\
\text { Coca }=13 \\
\text { Deca }=3\end{array}$ & $\begin{array}{c}\text { Micelle } 1 \\
\text { Sulf }=19 \\
\text { Coca }=22 \\
\text { Deca }=8\end{array}$ \\
\hline $\begin{array}{c}\underline{\text { Micelle 2 }} \\
\text { Sulf }=18 \\
\text { Coca }=19 \\
\text { Deca }=0\end{array}$ & $\begin{array}{c}\text { Micelle 2 } \\
\text { Sulf }=21 \\
\text { Coca }=17 \\
\text { Deca }=2\end{array}$ & $\begin{array}{c}\text { Micelle 2 } \\
\text { Sulf }=30 \\
\text { Coca }=27 \\
\text { Deca }=7\end{array}$ & $\begin{array}{c}\text { Micelle } 2 \\
\text { Sulf }=21 \\
\text { Coca }=18 \\
\text { Deca }=12\end{array}$ \\
\hline I & $\mathbf{J}$ & $\mathbf{K}$ & $\mathbf{L}$ \\
\hline $\begin{array}{l}\text { Micelle 1 } \\
\text { Sulf }=23 \\
\text { Deca }=0 \\
\text { Micelle 2 } \\
\begin{array}{l}\text { Sulf }=57 \\
\text { Deca }=0\end{array}\end{array}$ & $\begin{array}{l}\text { Micelle 1 } \\
\text { Sulf =26 } \\
\text { Deca }=1 \\
\text { Micelle 2 } \\
\begin{array}{l}\text { Sulf }=54 \\
\text { Deca }=4\end{array}\end{array}$ & $\begin{array}{c}\text { Micelle } 1 \\
\text { Sulf }=80 \\
\text { Deca }=10\end{array}$ & $\begin{array}{l}\text { Micelle 1 } \\
\text { Sulf }=21 \\
\text { Deca }=3 \\
\text { Micelle 2 } \\
\text { Sulf }=59 \\
\text { Deca }=17\end{array}$ \\
\hline $\mathbf{M}$ & $\mathbf{N}$ & $\mathbf{O}$ & $\mathbf{P}$ \\
\hline $\begin{array}{c}\text { Micelle } 1 \\
\text { Sulf }=40 \\
\text { Coca }=40 \\
\text { Deca }=0\end{array}$ & $\begin{array}{c}\text { Micelle } 1 \\
\text { Sulf }=40 \\
\text { Coca }=40 \\
\text { Deca }=5\end{array}$ & $\begin{array}{l}\text { Micelle } 1 \\
\text { Sulf }=40 \\
\text { Coca }=40 \\
\text { Deca }=10\end{array}$ & $\begin{array}{c}\text { Micelle } 1 \\
\text { Sulf }=40 \\
\text { Coca }=40 \\
\text { Deca }=20\end{array}$ \\
\hline
\end{tabular}

